Hautarzt 2011 · 62:240-240

DOI 10.1007/s00105-011-2139-4

Online publiziert: 23. Februar 2011

(c) Springer-Verlag 2011

Redaktion

J. Krutmann, Düsseldorf

Redaktionelle Mitarbeit

M. Berneburg, Tübingen

R. Gläser, Kiel

C. Hafner, Regensburg

B. Homey, Düsseldorf

B. Homey

Hautklinik, Heinrich-Heine-Universität, Düsseldorf

\title{
Inhaltsstoff von Kuhstallstaub schützt vor Allergien
}

voll gezeigt, dass pflanzliche Arabinogalactane aus Graspollen

- in hohen Konzentrationen in Kuhstallstäuben vorkommen,

- in murinen dendritischen Zellen die Produktion des immunmodulierenden Zytokins Interleukin-10 stimulieren,

- murine dendritische Zellen in der Art und Weise beeinflussen, dass in vitro keine allergischen Reaktionen induzierbar sind,

- nach intranasaler Applikation Mäuse vor der Entwicklung einer Sensibilisierung gegenüber einem Modellallergen und der Entwicklung von allergischen Entzündungsreaktionen der Lunge in Asthmamodellen schützen. der Ausbildung allergischer Erkrankungen schützt. Im Verlauf haben sich wissenschaftliche Studien intensiv mit der Charakterisierung von mikrobiellen Erregern und ihren Produkten in Kuhstallstäuben beschäftigt und protektive Wirkungen (z. B. für bakterielle Lipopolysaccharide) beschrieben. Neben bakteriellen Produkten, die häufig nur in geringen Konzentrationen in Kuhstallstäuben vorliegen, dominieren Substanzen, die vom pflanzlichen Futtermaterial ausgehen, die Kuhstallstaubzusammensetzung. Unter den pflanzlichen Produkten konnte eine Stoffklasse, sog. Arabinogalactane, in hoher Konzentration in Kuhstallstäuben nachgewiesen werden. Arabinogalactane sind Polysaccharide, denen eine immunmodulierende Funktion zugeschrieben wurde.

In einer aktuellen Publikation von Peters et al. [1] im Journal of Allergy and Clinical Immunology wurde nun eindruck-

\section{O In Kuhstallstäuben} vorkommende pflanzliche Arabinogalactane haben eine immunmodulierende Funktion

Zusammenfassend zeigen die Autoren erstmals das protektive Potenzial von pflanzlichen Arabinogalactanen in vivo und geben mit der Induktion von Interleukin-10 erste Hinweise auf den molekularen Wirkmechanismus. Die Produktion von Interleukin-10 alleine ist jedoch nicht ausreichend, um die schützenden Effekte von diesen pflanzlichen Polysacchariden zu erklären. Weitere Untersuchungen sind notwendig, um die molekularen und zellulären Mechanismen aufzudecken.

Unter realen Umweltbedingungen werden pflanzliche Arabinogalactane im Konzert mit bakteriellen Lipopolysacchariden (LPS) zusammenwirken und über Lectine und Toll-like-Rezeptoren immun- modulierende Effekte vermitteln. Bei Betrachtung der relativen Zusammensetzung von Kuhstallstäuben ist jedoch auffällig, dass die Konzentration von bakteriellen LPS deutlich niedriger ist als die Konzentration von pflanzlichen Arabinogalactanen. Abschließend ist es wichtig, darauf hinzuweisen, dass sich pflanzliche Arabinogalactane untereinander deutlich in der Struktur unterscheiden. Erste experimentelle Hinweise aus der Arbeit von Peters et al. verdeutlichen, dass der Struktur pflanzlicher Polysaccharide eine entscheidende Bedeutung zukommt.

\section{Fazit für die Praxis}

Diese Untersuchungen sind ein hervorragendes Beispiel dafür, wie hypothesengetriebene epidemiologische Forschung Fragestellungen für die experimentelle Forschung generiert und die Aufklärung von zugrunde liegenden Mechanismen schließlich Ansatzpunkte für die Entwicklung von Strategien in der Prävention und der Therapie von Allergien liefert.

\section{Korrespondenzadresse}

\section{Prof. Dr. B. Homey}

Hautklinik, Heinrich-Heine-Universität Moorenstr. 5, 40225 Düsseldorf

Bernhard.Homey@uni-duesseldorf.de

Interessenkonflikt. Keine Angaben.

\section{Literatur}

1. Peters $M$, Kauth $M$, Scherner $O$ et al (2010) Arabinogalactan isolated from cowshed dust extract protects mice from allergic airway inflammation and sensitization. J Allergy Clin Immunol 126:648656 